*6. Prescher A, Brors D. Metastases to the paranasal sinuses: case report and review of the literature. Laryngorhinootologie 2001; 80 (10): 583-94.

**7. Sabo R, Sela M, Sabo G, Herkovitz P, Feinmesser R. Metastatic hypernephroma to the head and neck: inusual case reports and review of the literature. $\mathrm{J}$ Otolaryngol 2001; 30 (3): 140-4.

8. Koscielny S. The paranasal sinuses as metastatic site of renal cell carcinoma. Laryngorhinootologie 1999; 78 (8): 441-4.

Casos Clínicos

Arch. Esp. Urol. 2010; 63 (2): 150-153

\section{ADENOCARCINOMA VESICAL DE CÉLULAS EN ANILLO DE SELLO: REVISIÓN DE NUESTROS CASOS ENTRE 1990-2009}

\section{Luis A. Busto Martín, Manuel Janeiro País, Juan}

González Dacal, Venancio Chantada Abal y Luis Busto Castañón.

Servicio de Urología. Complejo Hospitalario Universitario de Coruña. La Coruña. España.

Resumen.- OBJETIVOS: Presentar una revisión de los adenocarcinomas primarios de vejiga de células en anillo de sello de nuestro servicio.

\section{CORRESPONDENCIA}

Luis A. Busto Martín

Complejo Hospitalario Universitario de Coruña.

la Coruña. (España)

lbm@urologiabusto.com

Aceptado para publicar: 7 de octubre 2009
MÉTODO/RESULTADO: Revisamos las RTU de vejiga realizadas entre 1990 y 2009 encontrando 9 casos de tumores primarios de vejiga de células en anillo de sello (4 puros y 5 mixtos). 8 eran varones y 1 mujer, con edades comprendidas entre los 39 y los 82 años. Tratamiento definitivo consistió en cistectomía radical con derivación tipo Bricker en cuatro de los pacientes, cistectomía con derivación Mainz II en otro de ellos y paliativo en los cuatro restantes con RTU en tres casos y NPC paliativas en otro. Empleamos quimioterapia adyuvante en 3 casos. Sólo dos de los pacientes estaban vivos en el momento del estudio. La media de supervivencia fue de 327 días para los tumores puros y de 586 para los mixtos.

CONCLUSIÓN: Los adenocarcinomas primarios de vejiga con células en anillo de sello son unos tumores poco frecuentes, con peor pronóstico que los de células transicionales. Es importante que descartemos los posibles orígenes metastásicos (de estómago, próstata, ovario, pulmón) ya que el manejo a seguir será distinto en función de la localización del tumor primario. El tratamiento de elección en los primarios de vejiga es la cistectomía radical, con quimioterapia adyuvante en los casos que sea posible. La supervivencia a 5 años es $<$ al $11 \%$.

Palabras clave: Células en anillo de sello. Adenocarcinoma de vejiga. Tumor vesical.

Summary.- OBJECTIVES: To present a revision on the signet-ring cell bladder adenocarcinomas found in our department.

METHODS/RESULTS: We reviewed all the transurethral resections of the bladder (TURB) performed between 1990 and 2009 finding 9 cases of primary signet ring cell adenocarcinomas ( 4 pure and 5 mixed). Eight were male and one female, with ages between 39 and 82 years. Definitive treatment was radical cystectomy with Bricker's urinary diversion in four patients, cysctectomy with Mainz's II diversion in one patient and palliative management with TURB in three cases and percutaneous nephrostomy in the remaining case. We used adyuvant chemotherapy in three cases. Only two patients were alive at the time of the study. Mean survival was 327 days for pure tumors and 586 for the mixed ones.

CONCLUSIONS: Signet-ring cell primary adenocarcinoma of the bladder is an uncommon type of tumor, with worse prognosis than transitional cell cancer. It is important to discard other possible metastatic origins llike stomach, prostate, lung, or ovaryl because the management will be different. Radical cystectomy is the treatment of choice, with adyuvant chemotherapy if possible. Five year survival is less than $17 \%$.

Keywords: Signet-ring cell. Bladder adenocarcinoma. Bladder tumor. 


\section{INTRODUCCIÓN}

El adenocarcinoma vesical representa entre el 0,5 y $2 \%$ de los carcinomas vesicales.

Dentro de los adenocarcinomas vesicales, el $20 \%$ son de células en anillo de sello siendo además una de las variedades más agresivas, lo cual empeora su pronóstico con respecto a los carcinomas de células uroteliales debido a su crecimiento intramural y tardío diagnóstico.

El objeto de nuestro estudio es la revisión de los casos de adenocarcinoma vesical de células en anillo de sello puros y mixtos diagnosticados y tratados en nuestro servicio entre 1990 y 2009.

Incluimos aquellos tumores considerados como primarios de vejiga, que son más infrecuentes que los debidos a infiltración local desde órganos vecinos y los metastásicos.

\section{CASO CLÍNICO}

Se revisaron las historias clínicas de todos los pacientes intervenidos en nuestro servicio a los que se les realizó RTU vesical entre los años 1990-2009 descartando aquellos tumores que no cumplían los criterios histológicos, excluyendo los adenocarcinomas metastáticos o por infiltración local de la vejiga.

Encontramos nueve casos con carcinoma vesical de células en anillo de sello diagnosticados y tratados en nuestro servicio entre Enero de 1992 y Enero de 2009; ocho de los pacientes eran varones y sólo había una mujer, con de edades comprendidas entre los 39 y los 82 años, con una media de 69 años.

El debut clínico fue la hematuria en seis de los casos y clínica irritativa con polaquiuria y disuria importante en

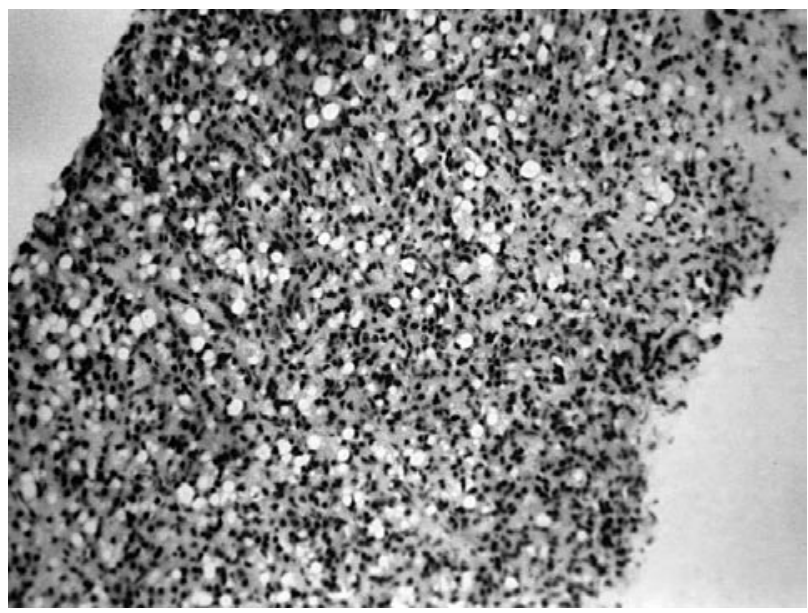

FIGURA 1. Anatomía patológica microscópica. uno de ellos. Uno de estos pacientes presentaba importante síndrome general con caquexia en el momento del diagnóstico.

El estudio citológico de la orina mostró células malignas en tres pacientes, atipias en dos y fue normal en los dos restantes.

La TAC mostró engrosamiento de la pared vesical en todos los casos, con infiltración del pericistio y adenopatías múltiples retroperitoneales en cuatro de los pacientes y uropatía obstructiva uni o bilateral en cuatro de los casos.

Dos de los casos presentaron alteración del estudio radiológico gastroduodenal siendo completado con endoscopia digestiva alta y baja con el hallazgo de gastritis crónica antral con focos de metaplasia intestinal en uno de los sujetos y pólipo adenomatoso de sigma en otro.

En todos los casos se realizaron estudios histológicos de las masas resecadas con inclusión en parafina, colorante Hematoxilina/Eosina y tinción PAS. En algunos casos se realizaron estudios inmunohistoquimicos (PSA, E-caderina, citoqueratina 20, S100) para descartar origen extravesical (1).

Tras el estudio anatomopatológico encontramos cuatro casos de adenocarcinoma vesical de células en anillo de sello primario puros y cinco mixtos con áreas de células en anillo de sello en el seno de carcinomas de células transicionales.

La anatomía patológica de estos tumores mostró células de citoplasma amplio eosinófilo con vacuola central PAS positiva y desplazamiento del núcleo hacia la periferia (morfología típica en anillo de sello) (Figura 1).

El tratamiento definitivo consistió en cistectomía radical con derivación tipo Bricker en tres de los pacientes,

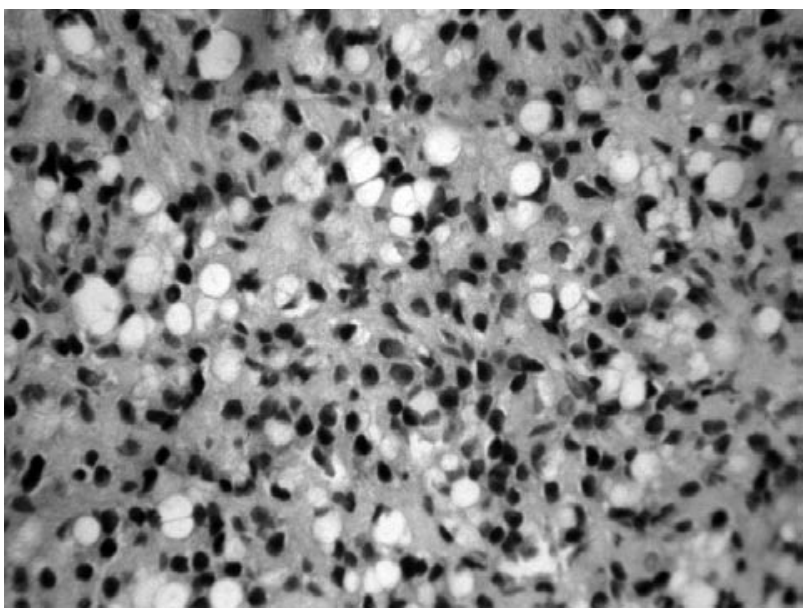

FIGURA 2. Anatomía patológica microscópica. 
cistectomía con derivación Mainz II en otro de ellos y paliativo en los cuatro restantes con RTU en tres casos y NPC paliativas en otro.

Recibieron quimioterapia adyuvante tres pacientes consistentes en MVAC.

La supervivencia fue de $570,480,250$ y 8 días para los tumores puros y de 512,410,280, 270 días y mayor de 4 años en un caso, para los tumores mixtos. Todos los pacientes, excepto dos habían fallecido en el momento de realizarse este estudio. En la literatura revisada la supervivencia global no supera el $11 \%$ a los 5 años $(2,3)$.

\section{DISCUSIÓN}

Dentro de los adenocarcinomas vesicales podemos distinguir tres subtipos histológicos según tengan un patrón glandular, coloide o de células en anillo de sello (1).

El adenocarcinoma vesical primario en células en anillo de sello es una neoplasia muy poco frecuente, habiéndose descrito unos 150 casos en la literatura mundial desde que Saphir describio en 1955 esta variedad de adenocarcinoma vesical (4-9)

Se trata de una variedad de adenocarcinoma vesical que tanto se puede presentar como forma pura o con más frecuencia de forma mixta, como focos de carcinoma en anillo de sello dentro de una neoplasia con predominio de células transicionales, siendo esta última la variedad más frecuente dentro de los primarios de vejiga.

Se han postulado entre sus posibles factores etiológicos la presencia de restos embrionarios, teoría que ya no se acepta al haberse detectado carcinomas en anillo de sello en el tracto urinario superior. Otra teoría sería la de una irritación crónica producida por infecciones recurrentes, extrofia vesical o la presencia de litiasis (10)

Una tercera teoría, que es la más aceptada hoy en día, es la aparición de la neoplasia a partir de células pluripotenciales de la mucosa vesical (11).

El debut clínico se suele producir en la década de los 50 - 60, siendo más frecuente en varones (En nuestra serie ocho varones y una mujer), siendo la hematuria el síntoma de inicio más frecuente ( $80 \%$ en nuestra serie), seguido por la clínica irritativa $(60 \%)$.

El pronóstico en los casos puros es extremadamente malo debido a su gran facilidad para metastatizar y la presencia de una enfermedad avanzada en la mayoría de los casos al diagnostico. La supervivencia media en nuestra serie para los casos puros fue de 327 días y de 586 para los tumores mixtos. Hay que señalar que cuando se trata de tumores mixtos el pronóstico es peor que si se tratase de un tumor similar pero solo de células transicionales puras $(2,12)$.

El tratamiento de elección es la cistectomía radical, pudiendo seguirse con quimioterapia adyuvante, aunque este tipo de tumores se caracterizan por una gran resistencia a los tratamientos quimioterápicos y radioterápicos. La quimioterapia paliativa se reserva para casos avanzados donde la cirugía ya no tendría valor (12).

En algunos casos la cistectomía parcial se ha empleado para tratar casos seleccionados como tumores presentes en cúpula o uracales de fácil resección con unos márgenes de seguridad de al menos $1-2 \mathrm{~cm}(1,13,14)$.

Una característica de estos tumores que suele dificultar su diagnóstico cistoscópico es la tendencia a crecer infiltrando la pared vesical en forma de linitis plástica

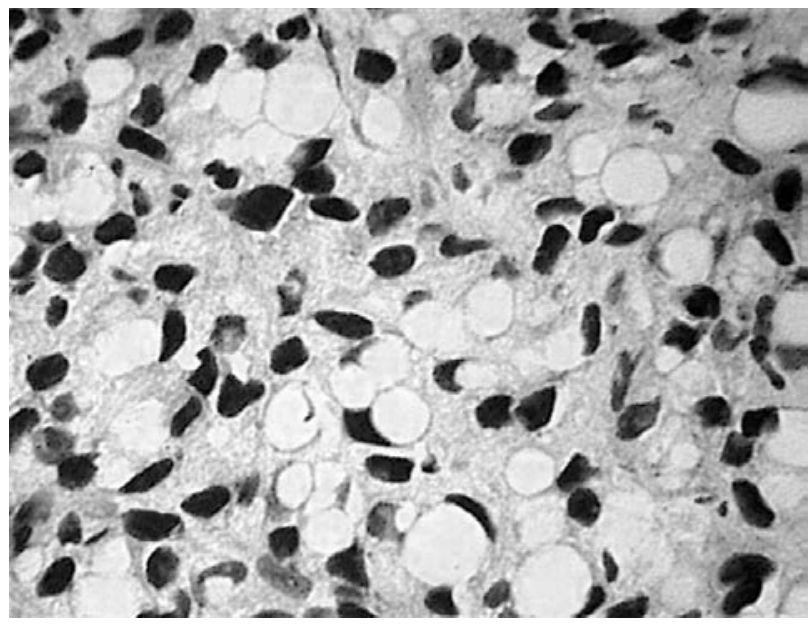

FIGURA 3. Anatomía patológica microscópica.

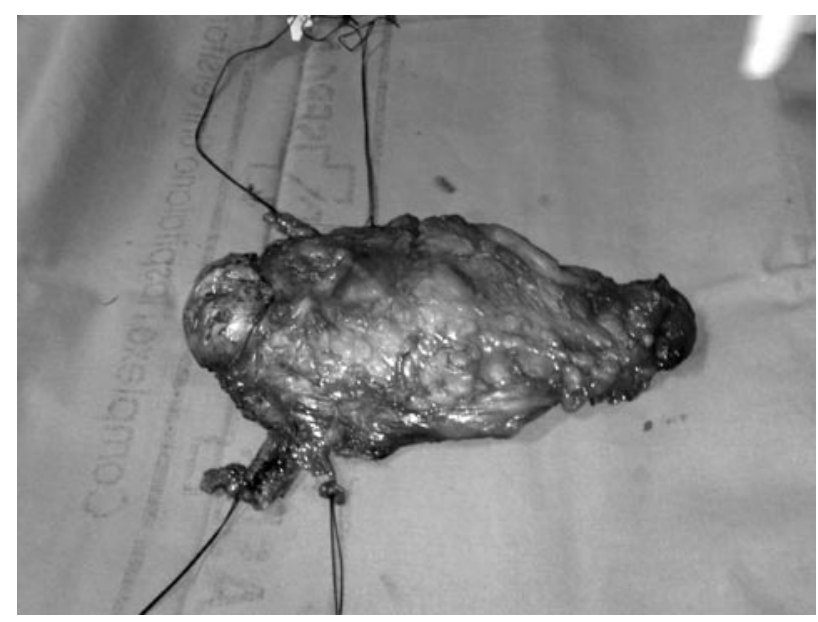

FIGURA 4. Pieza quirúrgica. 
infiltrando el espesor de la pared vesical bajo la mucosa, lo que puede retrasar su diagnostico y empeorar claramente su pronóstico al producir una mayor clínica irritativa y menos hematuria $(1,15)$.

\section{CONCLUSIONES}

Como conclusión hay que resaltar la rareza de los tumores vesicales primarios de células en anillo de sello, su alta agresividad y mala respuesta al tratamiento quirúrgico y médico, siendo los de peor pronóstico dentro de los adenocarcinomas vesicales. Hay que realizar diagnóstico diferencial con el posible origen extravesical del tumor (tracto digestivo, próstata, mama, ovario) para poderlos catalogar de primarios, ya que su enfoque terapéutico sería distinto. Por lo tanto estos pacientes deben ser estudiados de forma completa con las pruebas de imagen, endoscopia y biopsias necesarias para completar un correcto diagnostico.

\section{BIBLIOGRAFÍA y LECTURAS RECOMENDADAS ( ${ }^{*}$ lectura de interés $y^{* *}$ lectura fundamental)}

1. Cruz-GonzalesGH, Sanchez-Salas RE, Palmer-Roman KJ, Sanchez Rivero FR,Sanchez Salas R, Daboin I, et al. Adenocarcinoma en celulas en anillo de sello primario de vejiga. presentacion de un caso clinico con tratamiento multidisciplinario. Actas Urol Esp 2007;31(8):919-22.

2. Queipo Zaragozá JA, Chicote Pérez F, Borrell Palanca A, Beltrán Meseguer JF, Alcalá-Santaella Casanova C, Martínez García B, et al. Tumores vesicales inusuales: carcinoma epidermoide, adenocarcinoma y sarcoma primarios. Comportamiento clínico. Nuestra experiencia. Actas Urol Esp 2003, 27(2): 123-31.

3. Anderstrom C, Johansson SL, Schultz L. Primary adenocarcinoma of the urinary bladder. A clinicopathologic and prognostic study. Cancer 1983; 52: 1.273-80.
4. Fiter L, Gimeno F, Martin L, Gómez Tejeda L. Signet-ring cell adenocarcinoma of bladder. Urol, 1993;41(1):30-3.

5. Saphir O. Signet ring cell carcinoma of the urinary bladder. Am J Pathol. 1955;31(2):223-31.

6. Robert H, Young MD, John N, et al. Unusual forms of carcinoma of the urinary bladder. H path, 1998; 22: 948.

7. Fernández A, Maganto E, García R, Vallejo I, Escudero A. Adenocarcinoma vesical primario tipo células en anillo de sello. Arch Esp Urol, 1990; 43: 355-8.

8. Varo Solís C, Báez Perea JM, Garrido Insúa S, Beltrán Aguilar V. Neoplasia de células en anillo de sello. Una variedad histológica rara como tumor vesical primario. Actas Urol Esp, 2002; 26:297-301.

9. Fernández Fernández A, Maganto Pavón E, Vallejo Herrador J, Escudero Barrilero A.. Primary adenocarcinoma of the bladder as single tumor. Study of 7 cases and review of the literature. Actas Urol Esp. 1990;14(1):18-22.

10. Kumar PV, Youssefi A, Ahmad A. Primary signet ring cell adenocarcioma of the urinary bladder with calculi. Br J Urol, 1986;58(3):342-3.

11. Martinez Piñeiro L, Gonzalez Peramato P, Hidalgo de la Peña J, Cisneros J,Cozar JM, Martinez Piñeiro JA. Adenocarcinoma vescical primario: Estudio retrospectivo de 11 casos y revision del conjunto. Arch Esp Urol, 1991;44 (2).131-8.

12. Burnett AL, Epstein JI, Marshall FF. Adenocarcinoma of urinary bladder: classification and management. Urol, 1991;37(4):315-21.

13. Paul AB, Hunt CR, Harney JM, Jenkins JP, Mcmahon RF. Stage 0 mucinous adenocarcinoma of the urachus. J Clin Pathol, 1998;5(6):483-4.

14. Ojea Calvo A, Nuñez Lopez F, Dominguez Freire A, Alonso Rodrigo B, Rodriguez Iglesias J, Benavente Delgado JM, Barros Rodriguez et al. Adenocarcinoma mucinoso de uraco. Actas Urol Esp, 2003; 27(2)142-6.

15. Caballero Gómez M, Mariño del Real J, Simón Asuar Aydillo S, Abengozar García Moreno A, Mateos Blanco J, Murillo J. Adenocarcinoma vesical primario de células en anillo de sello. Portación de un caso clínico. Actas Urol Esp, 2000; 24 (9): 749-52. 